\title{
La descentralización de funciones sociales y la redefinición de los Estados locales. Acerca de la implementación de políticas sociales por parte del municipio de Santa Fe. Los casos del programa nacional Jóvenes con Más y Mejor Trabajo (JMyMT) y del programa local Los Solares (2008-2013)*
}

Florencia Inés Pretto*

\section{Resumen}

Este artículo presenta un estudio de caso que tiene como objetivo conocer la manera en que la Municipalidad de Santa Fe (período 2008-2013) asumió nuevas funciones sociales. El estudio se enmarca en los procesos de descentralización de funciones sociales que tuvieron lugar en la Argentina de los 90 y que concedieron una participación creciente a los Estados locales en la implementación de políticas sociales propias y nacionales. Las estrategias metodológicas utilizadas fueron de carácter cualitativo y estuvieron basadas en el análisis de documentos, discursos y entrevistas. Se realizó, en particular, un análisis del programa nacional Jóvenes con

\footnotetext{
* Artículo recibido el 14 de Agosto de 2015. Aceptado el 2 de Diciembre de 2015.

** El presente artículo tiene como finalidad exponer las principales conclusiones de una tesis de grado presentada en diciembre del 2014 en el marco de mi graduación como Licenciada en Ciencia Política en la Universidad Nacional del Litoral. Dirección de Tesis: Mg. María Jimena García Puente.

Universidad Nacional del Litoral. Santa Fe. Argentina.

Correo electrónico: florpretto@hotmail.com
} 
Más y Mejor Trabajo y del programa local Los Solares, ambos implementados por el municipio santafesino.

\section{Palabras clave}

Política Social - Descentralización - Estados locales.

\section{The decentralization of social functions and the redefinition of the local states. About the implementation of social policies by the municipality of Santa Fe. The cases of the national program Jóvenes con Más y Mejor Trabajo (JMyMT) and local program Los Solares (2008-2013)}

\section{Abstract}

This article presents as its main objective the way in which the local authorities of Santa Fe took over some new social functions (from 2008 to 2013). This survey frames the processes of decentralization of the social functions that were adopted in Argentina during the 90's. Owing to this, the local states were provided an increasing participation as regards the implementation of their own and national social policies. Methodological strategies, based on the analysis of documents, speeches and interviews, were used to approach a qualitative value. The focus of this survey was particularly set on the study of a national program called "Jóvenes con Más y Mejor Trabajo" and a local program called "Los Solares". Both of these programs were also implemented by the local authorities.

\section{Keywords}

Social policy - Decentralization - Local states. 


\section{Introducción}

En el presente artículo nos proponemos conocer cómo la Municipalidad de Santa Fe asumió nuevas funciones sociales (2008-2013) en el marco de la consolidación del proceso de descentralización de responsabilidades sociales llevado a cabo por el Estado Nacional argentino desde finales del siglo XX. En tal sentido, el problema abordado en este trabajo es el de la redefinición de los Estados locales argentinos y la conformación de renovados procesos de articulación entre estos últimos y el nivel nacional (Cormick, 1996). Específicamente, la problemática analizada hace referencia a la participación que en las últimas décadas han adquirido los municipios argentinos en la implementación de políticas sociales -ya sean nacionales, provinciales o municipales, y a su configuración como espacios complejos contenedores de diversos sistemas, de origen local y transferidos, organizados bajo la forma de servicios y programas destinados a la comunidad (Andrenacci, 2001).

En el mundo académico existen acuerdos generalizados en torno a la configuración que hubo en nuestro país de nuevos esquemas de distribución de responsabilidades (Cao, 2008), en donde a las tradicionales funciones municipales referidas principalmente a la regulación, construcción y mantenimiento de la estructura urbana, se le sumaron otras de carácter económico y social, como ser las políticas de empleo y promoción de la inversión, de generación de infraestructuras educativas y tecnológicas, de mejoramiento de la competitividad y promoción de redes productivas, entre otras (García Delgado, 1997). Estos procesos de redefinición de los Estados locales argentinos adquirieron formatos disímiles en función de las características de los territorios y de las capacidades institucionales y financieras de cada municipio (Repetto y Dal Masseto, 2003).

Cabe señalar que la situación problemática exhibida en los párrafos precedentes se enmarca en un proceso más amplio de reestructuración de la política social y económica argentina que se profundizó en los 90 y buscó racionalizar el gasto público y delimitar los ámbitos de intervención del Estado nacional, principalmente a través de la descentralización y privatización de la prestación de servicios universales de salud y educación (Oszlak, 2001). A propósito de la política social, a partir de la década de los 90, esta presentó variaciones en sus modos de "financiamiento, funcionamiento y administración, [así como] en su régimen de acceso y tipo de beneficios ofrecidos" (Hintze, 2003: 2). 


\section{¿Redefinición del municipio santafesino?}

Ante esta situación problemática y considerando nuestra inquietud inicial, definimos como objetivo general de este artículo el de (1) dar a conocer cómo la municipalidad de Santa Fe asumió, en el período 2008-2013, nuevas funciones sociales mediante el examen del programa social nacional Jóvenes con Más y Mejor Trabajo (PJMyMT) y del programa social municipal Los Solares, ambos implementados por el municipio. Específicamente, nos proponemos (1a) examinar la articulación entre el municipio y el nivel nacional, y el grado de autonomía decisional del primero durante la implementación del programa JMyMT. Como segundo objetivo específico buscamos (1b) reconocer y analizar las estrategias que fueron utilizadas por el municipio para implementar los programas sociales seleccionados.

Entendemos que los resultados y conclusiones de la investigación realizada constituyen un aporte a la bibliografía existente sobre los procesos de descentralización de funciones sociales y de emergencia de nuevos Estados locales que se profundizaron en la Argentina de los 90 y se mantienen en la actualidad. Retomando a Badía y Carmona (2008), los resultados alcanzados permiten "discutir experiencias de gestión local que incorporen la perspectiva de articulación intergubernamental (...) [y así] avanzar en la construcción de marcos teóricos y metodológicos que posibiliten la consolidación de este campo de estudio, necesario para dar cuenta de buena parte de los procesos sociales que se han profundizado en los últimos años" (Badía y Carmona, 2008: 11). De esta manera, el presente artículo se suma a los estudios de caso del municipio santafesino desde un aporte centrado en el análisis de las políticas sociales y de la articulación sostenida con el Estado Nacional.

Con relación al período abordado, se tomaron los años 2008-2013 -gestiones de los intendentes Mario Barletta y José Manuel Corral- debido a que estos representan un punto de inflexión en la dirigencia partidaria del gobierno de la ciudad de Santa Fe, luego de 16 años de gestión del partido justicialista. Además, la cercanía temporal tornó viable el acceso a la información y datos requeridos para la investigación, así como la coordinación de entrevistas con los actores claves.

Es necesario mencionar que la metodología utilizada fue de carácter cualitativo y estuvo basada en el análisis de documentos, discursos y entrevistas. El corpus analizado estuvo integrado por documentos de carácter oficial: decretos y normativa de los Estados nacional y municipal, destinados a la divulgación, reglamentación y contextualización de los programas sociales seleccionados. Además, el corpus incluye los discursos proferidos por los intendentes Mario Barletta y José Manuel Corral durante la asunción de sus respectivos cargos y la inauguración de las sesiones 
ordinarias del Honorable Concejo Municipal (HCM). Por último, aunque de vital importancia, el corpus contiene entrevistas de carácter semiestructurado que estuvieron dirigidas a funcionarios locales y nacionales claves cumpliendo funciones en el territorio santafesino.

\section{Características de la ciudad de Santa Fe y del municipio bajo estudio ${ }^{2}$}

La ciudad de Santa Fe fue fundada en el año 1573 y es la capital de la provincia homónima. Su población asciende a 391231 habitantes, registrando una tasa anual de crecimiento de $0,7 \%$ con respecto al censo del año 2001. Al inicio del periodo de gobierno del intendente Mario Barletta, la ciudad presentaba un 9,4\% de desocupación y un $23,3 \%$ de personas pobres.

En cuanto a la Municipalidad de Santa Fe, esta tuvo origen en el año 1861, décadas después de que la Provincia de Santa Fe naciera como unidad territorial autónoma e independiente de Buenos Aires. En la actualidad, el régimen municipal santafesino constituye uno de los pocos Estados locales argentinos sin autonomía institucional, es decir, sin facultad para ejercer el poder constituyente mediante el dictado de una carta orgánica municipal (Costamagna, 2007). Desde el retorno de la democracia en Argentina, en 1983, el municipio de Santa Fe fue presidido por el partido Peronista (PJ). ${ }^{3}$ A partir del año 2007 hasta la actualidad, se inició un período de cambio en la gobernación del Ejecutivo santafesino con la asunción de los partidos de la Unión Cívica Radical (UCR) y del Frente Progresista Cívico y Social (FPCyS). Desde entonces, el municipio se encuentra organizado en ocho Distritos (Ordenanza No 11513/08) a través de los cuales se busca descentralizar la prestación de servicios municipales en los distintos barrios de la ciudad.

\section{La cuestión social en el municipio de Santa Fe: los programas Jóvenes con Más y Mejor Trabajo y Los Solares}

El programa JMyMT se anuncia y reglamenta en el año 2008 por medio de las resoluciones No 497/08 y 261/08 de la Secretaría de Empleo (SE) del Ministerio de Trabajo, Empleo y Seguridad Social (MTEySS) de la Nación. Mediante este programa, se

\footnotetext{
${ }^{2}$ El presente apartado se elaboró en base a la información estadística que brinda el Instituto Provincial de Estadísticas y Censos (IPEC) mediante el Censo de Población, Hogares y Viviendas de 2010 y la Encuesta Permanente de Hogares (EPH). Para los niveles de desempleo y pobreza específicamente, se utilizaron datos del año 2007 cuando asume el intendente Mario Barletta.

${ }^{3}$ A excepción del período 1989-1991 en donde presidió el municipio el por entonces Partido Demócrata Progresista (PDP).
} 
busca "generar oportunidades de inclusión social y laboral de las y los jóvenes a través de acciones integradas que les permitan identificar el perfil profesional en el cual deseen desempeñarse, finalizar su escolaridad obligatoria, realizar experiencias de formación y/o de prácticas calificantes en ambientes de trabajo, iniciar una actividad productiva de manera independiente o insertarse en un empleo" (Resolución $N^{\circ}$ 497/08).

El PJMyMT se crea en el marco del Plan Integral para la Promoción de Empleo (Resolución N 256/03) presentado en el año 2003 por el MTEySS de la nación. Dicho plan se encuentra en vigencia y busca contribuir a la generación, sostenimiento y mejora del empleo mediante la articulación de distintas políticas públicas implementadas por las áreas competentes del MTEySS, y de los distintos órganos del gobierno nacional, provincial y municipal. A través de este plan, el MTEySS promueve en provincias y municipios el establecimiento de Acuerdos Territoriales para la Promoción de Empleo dirigidos a formular e implementar propuestas que, desde un enfoque estratégico e integral, den respuesta a los problemas de empleo y trabajo de la población. Así, en función de lo establecido en este plan en el año 2006, la municipalidad de Santa Fe y el MTEySS de la nación firmaron el Convenio Marco $\mathrm{N}^{\circ}$ 96/06 para "desarrollar de manera permanente estrategias locales de promoción del empleo en el territorio municipal, en articulación con los sectores públicos y privados relacionados con la producción, la formación y el empleo". La municipalidad de Santa Fe comienza a implementar en el año 2010 el PJMyMT a través de un Protocolo Adicional al Convenio Marco (Protocolo Adicional n 8).

Por su parte, el programa Los Solares se crea en el año 2009 por medio del Decreto municipal No 00725/09 de la Secretaría de Gobierno de la Municipalidad de Santa Fe, y se ubica dentro del Plan Social Familias Incluidas. Su principal objetivo es el de "abordar desde las políticas públicas locales la promoción y protección de los derechos de niños, niñas y adolescentes en condiciones de exclusión y vulnerabilidad social, desde una perspectiva integral y participativa que involucre al grupo familiar y al entorno barrial" (Decreto Nº 00725/09). Para alcanzar tal objetivo se buscó transformar a los antiguos comedores municipales en Centros de Desarrollo entendidos como espacios de "encuentro, sociabilidad e integración de niños y adolescentes (y sus grupos familiares) en situación de exclusión y vulnerabilidad social". Además de diseñar, impulsar y coordinar acciones que promuevan los derechos de niñas, niños y adolescentes, con este programa el municipio también promovió estrategias conjuntas con los diversos niveles del Estado que operan en el territorio y con diversas organizaciones de la sociedad civil involucradas en la temática. 
El órgano responsable directo de la implementación de los dos programas es la Secretaría de Desarrollo Social de la Municipalidad de Santa Fe. En el caso del PJMyMT, este depende directamente de la nueva Subsecretaría de Empleo de la Secretaría de Desarrollo Social, la cual dirige y coordina las actividades realizadas en la Oficina de Empleo municipal. Por su parte, la coordinación general del programa Solares está a cargo de la Subsecretaría de Acción Social de la Secretaría de Desarrollo Social. En ambos casos la implementación del programa requiere de la coordinación con otras Secretarías del Estado local que están involucradas en el abordaje de los problemas sociales del territorio santafesino.

Al ser el PJMyMT de origen nacional, su implementación también involucra a diversos órganos del MTEySS de la nación. De esta manera, la municipalidad de Santa Fe coordina su accionar con la Gerencia de Empleo y Capacitación Laboral (GECAL) de la SE del MTEySS de la nación situado en la capital santafesina. El ministerio cuenta con dependencias de la GECAL en diversos puntos del país -en las principales ciudadescon el propósito de descentralizar sus políticas y estar en contacto directo con las distintas realidades locales del territorio argentino. ${ }^{4}$

\section{Las nuevas funciones sociales del Estado local santafesino}

En términos generales, (1) podemos afirmar que se constataron indicadores que dan cuenta de una preocupación del Estado local santafesino en la implementación de políticas sociales propias y nacionales. Ello se evidencia en la clara exposición de objetivos sociales por parte del municipio en sus planes de gobierno (Plan Desarrollo Santa Fe Ciudad y Plan Santa Fe 2020). Estos últimos se materializaron en la creación de nuevas secretarías en el organigrama municipal; en la revitalización y creación de

\footnotetext{
${ }^{4}$ Desde principios del siglo XXI, el MTEySS lleva adelante procesos de institucionalización y territorialización de políticas de trabajo y empleo nacionales (Madoery, 2011). Una de estas políticas fue la consolidación de las ya existentes Delegaciones Territoriales dependientes de la Secretaría de Trabajo (ST), y de las Gerencias de Empleo y Capacitación Laboral (GECAL) dependientes de la SE, específicamente de la Dirección Nacional de Servicio Federal de Empleo. Mientras las primeras se dirigen a controlar el trabajo en negro y a mediar en los conflictos laborales, las segundas buscan "garantizar la ejecución articulada de las políticas, planes, programas y acciones para promover el empleo, la capacitación laboral (...) y la empleabilidad de los trabajadores de su jurisdicción" (Resolución MTEySS N¹00/2012). Es por ello que la GECAL localizada en la ciudad de Santa Fe es el ente nacional con mayor responsabilidad en el territorio en lo tocante a la reglamentación y puesta en marcha del PJMyM.
} 
consejos (de la Mujer, de la Niñez y Adolescencia, Económico y Social, de Adultos Mayores, etc.); en la firma de convenios con otros niveles del Estado y en la ejecución de programas sociales dirigidos a alcanzar mayores grados de prevención e inclusión social en el territorio santafesino.

En relación con los objetivos de carácter social definidos por el municipio, durante el período analizado, la municipalidad de Santa Fe se propuso construir una ciudad inclusiva, justa y fraternal, dirigida a fomentar la cohesión social, a proteger y promover la salud y a garantizar el acceso igualitario a la educación y a la cultura (Barletta, 2007). El municipio aseguró alcanzar estos niveles de inclusión por medio de la incorporación del conjunto de la sociedad al entramado social y productivo, y del fortalecimiento de las condiciones reales de autonomía, equidad y calidad de vida. En este marco, afirmó también la necesidad de alcanzar una ciudad más integrada a través del accionar conjunto de las distintas secretarías de gobierno (Corral, 2012). En relación con esto último, la municipalidad concibió a la inclusión social como un objetivo que debe estar presente en todas las políticas de Estado y materializarse tanto en las obras públicas en diversos barrios de la ciudad, como en la promoción del empleo, en el fomento a la cultura y la salud, y en la ampliación de los niveles educativos de la ciudad (Corral, 2011). Sin embargo, el municipio santafesino reconoció la necesidad de implementar políticas sociales focalizadas en los sectores más vulnerables de la ciudad (Corral, 2014), y se propuso para ello "desarrollar políticas públicas inclusivas que hagan eje en la persona, en la familia y en los grupos sociales vulnerables, con vistas a disminuir la profundidad y diversidad de la exclusión y la pobreza" (Municipalidad de Santa Fe, 2008: 21).

Estos objetivos esbozados por el municipio se materializaron en el anuncio y ejecución de diversos programas sociales -con recursos propios y de origen nacional- pertenecientes a distintas áreas de gobierno.

Por su parte, la creación de la Secretaría de Desarrollo Social ${ }^{5}$ en reemplazo de la anterior Secretaría de Promoción Comunitaria encarnó gran parte de lo que fue la

\footnotetext{
${ }^{5}$ La creación de la Secretaría de Desarrollo Social tuvo lugar a principios del año 2008,durante la gestión del intendente Mario Barletta. Sin embargo, dicha creación se anuncia a finales del año 2007 por medio de la Ordenanza $n^{\circ}$ 11.437/07, en reemplazo de la anterior Secretaría de Promoción Comunitaria. En sus inicios, la Secretaría de Desarrollo Social de la Municipalidad de Santa Fe se componía de las Subsecretarías de Acción Social, Ambiente, Salud y Educación. Actualmente, estas dos últimas se transformaron en secretarías independientes y la Secretaría de Desarrollo Social está constituida por las Subsecretarías de Acción Social, Ambiente y Empleo (Ordenanza n 11829/12).
} 
asunción de nuevas funciones sociales por parte de la municipalidad de Santa Fe. Por medio de esta, el Estado local se propuso, entre otros fines, "formular políticas sociales que favorezcan la inclusión social, fortalezcan los procesos de prevención, asistencia y promoción, y el restablecimiento de los vínculos laborales, sociales, familiares y comunitarios" (Ordenanza No 11.437/07). Además, para una mayor organización en la manera de alcanzar estos propósitos, se anunció ante la comunidad el Plan de Desarrollo Santa Fe Ciudad, y, cuatro años más tarde, el Plan de Desarrollo 2020. En estos planes de gestión la municipalidad ordenó y agrupó sus actividades en función de las problemáticas a solucionar en la ciudad: ineficiencia y falta de transparencia en la administración pública; producción y desempleo; pobreza y bajos niveles de educación; medio ambiente e infraestructura; cultura, etc. Estos planes facilitaron al municipio la posterior toma de decisiones y la comunicación a la sociedad de los objetivos perseguidos y las herramientas para alcanzarlos (Corral, 2014).

En relación con esto último, el compromiso asumido por la municipalidad de Santa Fe en la admisión de nuevas funciones sociales también se reflejó en la constante redefinición y reorganización de su organigrama funcional y de los programas sociales implementados, transformaciones que se dieron acordes a los cambios producidos en las demandas del territorio. En relación con ello, en lo que respecta a las políticas sociales de empleo, en el año 2012 se creó la Subsecretaría de Empleo dentro de la reciente Secretaría de Desarrollo Social. Esta reemplazó a la Dirección de Trabajo y Empleo creada en el año 2008 y a través de ella se buscó nuclear a todos los programas de empleo vigentes. Con anterioridad, los escasos programas de empleo existentes eran implementados directamente desde la intendencia y/o a través de la Secretaría de la Producción. Otros ejemplos están dados por la reubicación de determinados programas sociales en otras dependencias o secretarías, y la conversión de subsecretarías en secretarías independientes y con mayor jerarquía. Ejemplo de este último caso es la transformación, en el año 2012, de las subsecretarías de Educación y Salud en Secretarías.

Por su parte, la creación del Programa de Descentralización y Gestión Territorial (Ordenanza No 11513/08) también contribuyó a la institucionalización de la política social en la ciudad de Santa Fe. Entre otros fines, a través de tal medida se buscó alcanzar una mayor eficacia y cercanía territorial en la implementación de políticas públicas, y mejorar los niveles de participación vecinal en la toma de decisiones sobre los problemas de cada barrio.

Como mencionamos anteriormente, para afrontar los problemas sociales de la ciudad, el municipio también recurrió a políticas de creación y revitalización de espacios de concertación. Con el Decreto № 00172/2012 se instauró un Gabinete 
Social dirigido a conformar un sistema integral de políticas sociales por medio de la "generación de una instancia estratégica que tenga bajo su órbita la responsabilidad de coordinar, establecer las prioridades y articular las políticas sociales a implementar", con énfasis en la familia y bajo los principios de integración, articulación, equidad y solidaridad. Asimismo, la municipalidad de Santa Fe creó nuevos consejos vinculados a la temática de lo social, y revitalizó y fortaleció otros preexistentes, todos ellos dirigidos a conformar un espacio de diálogo y coordinación en el tratamiento e implementación de las políticas sociales. De esta manera, además del Consejo de la Niñez y Adolescencia, actualmente la municipalidad de Santa Fe cuenta con un Consejo Consultivo de Mujeres (Ordenanza 11362); con un Consejo Económico y Social (Decreto $n^{\circ}$ 00466/09); Consejo de Educación -que solo abarca a sectores e instituciones locales-; Consejo de Salud -que se articula con otras jurisdicciones, principalmente con el Estado provincial, y organizaciones sociales del territorio-; Consejo de Adultos Mayores (ordenanza 11107); Consejo de Niños y Niñas (Decreto $n^{\circ}$ 1026/08) -que promueve la participación ciudadana de los niños en la definición y solución de los problemas de la ciudad); Consejo del Deporte, entre otros.

Por último, la firma de diversos convenios con el Estado nacional, principalmente con el MTEySS, también es reflejo del grado de compromiso asumido por el municipio en su admisión de nuevas funciones sociales. Si bien los espacios de diálogo y el grado de vinculación entre ambas esferas de gobierno varían en función de cuáles sean las temáticas y los actores intervinientes, en diversas ocasiones el municipio santafesino se alineó con la jurisdicción nacional a los fines de acceder a determinada información y, principalmente, a ciertos fondos económicos y financieros (Entrevista $\mathrm{n}^{\circ}$ 6). La municipalidad de Santa Fe adhirió a determinados programas nacionales debido a que concibe a estos últimos como una herramienta externa que le brinda mayores posibilidades y capacidades de gestión -mayor cantidad de prestaciones dirigidas a la sociedad, y de recursos humanos y financieros-, y que en ocasiones es utilizada para alcanzar objetivos sociales que van más allá de los definidos por cada programa nacional en particular (Entrevistas $n^{\circ} 4$ y 6 ). El municipio entendió que debía "coordinar esfuerzos y acciones, buscando las sinergias que pongan en acción los recursos con los que cada jurisdicción cuenta, con vistas a avanzar en un camino que trascienda un período de gobierno en función de acuerdos básicos a largo plazo" (Municipalidad de Santa Fe, 2008: 4). Asimismo, para la municipalidad de Santa Fe, todo acuerdo establecido con las jurisdicciones superiores debe respetar las decisiones y proyectos de ciudad resueltos en el nivel local, entendiendo que los Estados provincial y, principalmente, nacional, deben ser sensibles a las necesidades de la población santafesina (Corral, 2012 y 2013). 
A modo de resumen, consideramos que el conjunto de estas medidas constituye un importante avance en la institucionalización de la política social municipal. Sostenemos, a su vez, que la municipalidad de Santa Fe representa un caso concreto de refuncionalización de las administraciones locales argentinas, puesto que incorporó a sus responsabilidades y funciones de alumbrado, barrido y limpieza ( $A B L)$ otras de carácter económico y social.

\section{Relación entre la municipalidad de Santa Fe y el MTEySS de la nación en la implementación del PJMyMT: ¿autonomía del municipio santafesino?}

Articulación según la normativa:

Respecto a la articulación intergubernamental entre el municipio y el Estado nacional durante la implementación del programa JMyMT $\left.\mathbf{( 1}^{\mathbf{a}}\right)$, podemos afirmar que prevaleció una clara y respetada división de tareas entre ambas jurisdicciones que se sustentó en una abundante normativa de carácter nacional y municipal. Fue únicamente en situaciones puntuales en donde esta vinculación cordial se alteró. Esta buena articulación se evidencia en las resoluciones que a nuestro criterio funcionan como antecedentes y como facilitadoras de la relación interjurisdiccional. Concebimos como antecedentes institucionales y legales directos del proceso de articulación entre la municipalidad y la GECAL del MTEySS al proceso de creación e incorporación de la Oficina de Empleo de Santa Fe a la Red Federal de Servicio de Empleo ${ }^{6}$ (2005), y a la implementación de programas nacionales de empleo por parte de dicha oficina a partir del año 2006. También identificamos guías para la articulación durante la implementación del Jóvenes, al Convenio Marco 96/06 firmado por ambas esferas de gobierno, a los Protocolos Adicionales a dicho Convenio y a las resoluciones nacionales que anuncian y reglamentan el programa (Resoluciones No 497/08 y No 261/08 del MTEySS).

El Estado santafesino creó la Oficina de Empleo Municipal en el año 2005 (Resoluciones No 112017/05 del HCM y No 813/05 de la Secretaría de Promoción

\footnotetext{
${ }^{6}$ En el marco del Plan Integral para la Promoción de Empleo (2003), en el año 2005 la SE del MTEySS creó en el ámbito de la Dirección Nacional del Servicio Federal de Empleo una Red Federal de Servicio de Empleo (Resoluciones No 316/05 y 176/05), conocida también como Red de Oficinas de Empleo Municipales. Con esta Red se buscó nuclear y vigorizar a las Oficinas de Empleo Municipales que ya tenían convenio con el MTEySS. Además de fortalecer a las oficinas existentes, se instó a los municipios para la creación de nuevas con el fin de "consolidar espacios institucionales que permitan garantizar la prestación de servicios de empleo" (Resolución No 176/05).
} 
Comunitaria) y los responsables de su funcionamiento fueron la antigua Secretaría de Promoción Comunitaria y la actual Secretaría de la Producción. Con esta oficina, el municipio buscó "generar un espacio a fin de administrar y optimizar los recursos que aporta el Estado nacional y garantizar una mayor transparencia, tanto en las acciones de capacitación como de ejecución de los proyectos, tendientes a asegurar una racional distribución de las ayudas económicas a los beneficiarios de los programas emanados del MTEySS y desocupados en general" (Resolución № 813/05). De esta manera, el funcionamiento de la Oficina instauró en el municipio nuevos mecanismos institucionales de articulación entre algunas de sus secretarías y, lo que aquí importa, entre la propia municipalidad y la SE del MTEySS de la nación.

Asimismo, los programas nacionales de empleo implementados por esta oficina a partir del año 2006, principalmente el de Seguro de Capacitación y Empleo (Resolución No 336/06 Protocolo Adicional No 1 al Convenio Marco 69/06), constituyeron un punto de inflexión en los modos de concebir y operar la política de empleo nacional y local, en donde las políticas nacionales asistenciales y subsidiarias implementadas por el municipio -como el caso del Plan Nacional Jefes y Jefas de Hogar Desocupados (JJHD-Decreto No 565/02 programa)- cedieron protagonismo a políticas de capacitación y generación de puestos de trabajo (Entrevistas $n^{\circ} 1$ y 2 ).

\section{$\underline{\text { La articulación según los actores }}$}

Además de la normativa anunciada, la clara y respetada división de tareas se verifica en los buenos resultados que, de acuerdo a los actores entrevistados, se visualizaron durante la ejecución del programa, estos referidos al grado de comunicación, coordinación y respeto alcanzado entre los niveles. A pesar de ello, también se observaron situaciones de incomunicación que se evidenciaron en las entrevistas. Esto puede deberse a la ausencia de acuerdos entre los niveles para la creación de instituciones coordinadoras y al escaso conocimiento de la composición orgánica y programática presentado por cada jurisdicción con respecto a la otra. En otras palabras, consideramos que la puesta en marcha de instituciones dirigidas a dinamizar y organizar la interacción entre los niveles del Estado, junto a un mayor conocimiento recíproco de los organigramas funcionales y de las agendas de gobierno, constituirían avances en el proceso de articulación intergubernamental entre la municipalidad de Santa Fe y la GECAL del MTEySS de la nación.

Según las referentes del PJMyMT (Entrevista $n^{\circ} 1$ ), los procesos de articulación entre la GECAL y el municipio vislumbraron una intensa voluntad política y una interdependencia constante que se dio en términos comunicativos y de retroalimentación de resultados e información, lo cual no habría sido posible de 
alcanzar si no hubieran existido acuerdos previos que plasmaran de manera clara los criterios y objetivos generales y específicos a seguir por cada jurisdicción (Protocolo Adicional $\mathrm{N}^{\circ}$ 8). Asimismo, las coordinadoras del programa sostuvieron que para alcanzar esta buena articulación fue a su vez necesario que las metas compartidas entre ambas jurisdicciones estuvieran incluidas en sus respectivas agendas de gobierno, para que "si a una jurisdicción le va bien, a la otra también" (Entrevista $n^{\circ} 1$ ). Según las entrevistadas, los resultados del programa nacional se evalúan en función de los logros alcanzados en cada territorio, y si estos últimos son buenos es porque la coordinación entre ambas esferas fue óptima (Entrevista $n^{\circ} 1$ ).

Además, también podemos afirmar que el grado de autonomía decisional del municipio al momento de implementar el programa JMYMT es elevado $\mathbf{( 1}^{\text {a }}$ ). Ello se manifiesta en la posibilidad que tiene el Estado local de adaptar los objetivos del programa nacional al contexto local de implementación, es decir, a las características distintivas del territorio santafesino.

Si bien el municipio debe respetar y actuar en función de los objetivos y de ciertos requisitos legales y administrativos del programa, cuenta con poder de decisión para definir cómo presentar el programa ante la comunidad -la articulación con otros programas locales, el énfasis en determinadas prestaciones por sobre otras, el ofrecimiento de servicios por finalidad y no por programa, etc.- y cómo incorporarlo a su agenda y organigrama local -la creación de la Subsecretaría de Empleo, la contratación de asistentes sociales por fuera de los recursos nacionales, la coordinación con otras secretarías, entre otras iniciativas. Recordemos que, de acuerdo a referentes del programa nacional, la municipalidad de Santa Fe "tiene la potestad suficiente para ampliar y fortalecer aún más las herramientas provenientes desde nación" (Entrevista $\left.n^{\circ} 2\right)$.

En relación con los compromisos asumidos por el municipio, resulta de interés destacar la similitud con la que los referentes municipales conciben el alcance de la política de empleo local. Tanto la coordinadora del actual Programa Oficina de Empleo como el Secretario de Desarrollo Social entienden que las posibilidades del municipio en lo tocante al empleo son las de mejorar las condiciones de empleabilidad de los individuos. Lejos de poder generar fuentes de trabajo -política macroeconómica que incumbe al Estado nacional-, la tarea municipal debe centrarse y reducirse a la gestión de tareas de intermediación laboral, por ejemplo, vincular las necesidades del mercado local con la población desocupada santafesina. Lo que busca la gestión municipal es que "un ciudadano que se presenta en la Oficina de Empleo sea más empleable" (Entrevista $\left.\mathrm{n}^{\circ} 4\right)$. 


\section{Las estrategias locales de implementación de los programas sociales Jóvenes con Más y Mejor Trabajo y Los Solares}

A partir de los resultados obtenidos en nuestra investigación, afirmamos que la municipalidad de Santa Fe recurrió a variadas estrategias de implementación al momento de ejecutar el programa nacional JMyMT y el programa local Los Solares (1b). En primer lugar, ello se evidencia en la decisión de adecuar su agenda y organigrama funcional a los objetivos de los programas. En el caso del programa JMyMT, para su implementación se creó la Dirección de Empleo y Trabajo en dependencia del Ejecutivo, que luego fue transformada en la Subsecretaría de Empleo de la Secretaría de Desarrollo Social; se modernizaron y descentralizaron las Oficinas de Empleo; y se contrataron asistentes sociales para alcanzar un seguimiento mayor de los jóvenes desocupados. Asimismo, se reorganizó la oferta de la Oficina de Empleo y se presentó el programa Jóvenes junto a otros de carácter nacional y en menor medida local, a través de una matriz unificada de distintos servicios de empleo hacia la comunidad. Concretamente, para alcanzar los objetivos perseguidos por la Subsecretaría de Empleo, la municipalidad creó el programa Oficina de Empleo (D.M.M. No 00295/12) con el cual apuntó al "diseño de políticas integrales de empleo adecuadas a las posibilidades socioproductivas del territorio y acordes a los recursos disponibles del MTEySS y del gobierno local" (Subsecretaría de Empleo, 2013).

A propósito del programa Solares, para su ejecución se decidió crear un área municipal encargada de la coordinación del programa, a la vez que la dependencia funcional de este último pasó desde la antigua Subsecretaría de Educación hacia la Subsecretaría de Acción Social. Al igual que en el caso del PJMyMT, también se contrataron asistentes sociales para alcanzar un mayor acompañamiento de los niños, adolescentes y familias que asisten a los Centros de Desarrollo.

El énfasis colocado en las políticas de articulación intersectorial es otra de las estrategias de implementación utilizada por el municipio durante la gestión de los dos programas. Ello se manifiesta en los procesos de vinculación establecidos entre la Secretaría de Desarrollo Social y sus respectivas Subsecretarías de Acción Social y de Empleo, y las Secretarías de Producción, Cultura, Salud y Educación. Esta articulación intersectorial posibilita a su vez una determinada vinculación entre los distintos programas del gobierno municipal. En relación con esto último y de acuerdo a los resultados de nuestra investigación, podemos afirmar que la articulación programática es una característica distintiva del modo de gestión que propone la municipalidad para abordar de manera integral los problemas sociales que aquejan al territorio. 
Otra estrategia a la que recurrió el municipio al momento de implementar los programas fue la de utilizar las prestaciones de programas nacionales en la oferta de programas sociales locales. Cabe destacar que estos procesos de colocación de nombres propios a etapas de implementación de iniciativas nacionales, en determinadas ocasiones fueron llevados a cabo sin advertir sobre ello de manera explícita a la comunidad. ${ }^{7}$ En relación con el PJMyMT, la municipalidad de Santa Fe utilizó los recursos y esquemas organizacionales de este para la puesta en práctica de programas de origen local. Son casos paradigmáticos el anuncio de los programas Calles Seguras e Infancias Protegidas (Decreto DMM No 01948/13) y Fortalecimiento de Empleabilidad: Confección de Blancos Uniformes (Programa Yo Puedo). En el caso del programa Calles Seguras e Infancias Protegidas gestionado por las Subsecretarías de Acción Social y de Empleo de la SDS, el municipio ofreció a los individuos de 18 a 24 años las prestaciones de Inserción Laboral y Entrenamiento para el Trabajo que forman parte del PJMyMT (Subsecretaría de Empleo, 2013). Por su parte, el Programa Fortalecimiento de Empleabilidad es gestionado por la Subsecretaría de Empleo y la Secretaría de Educación, y, entre otros servicios, ofrece a sus beneficiarias de entre 18 y 24 años la posibilidad de inscribirse al programa nacional JMYMT (www.santafeciudad.com y Diario Uno).

Entendemos que esta manera de ofrecer las prestaciones del PJMyMT a la comunidad santafesina presenta compatibilidades con la concepción que los actores locales poseen del programa. Recordemos que, para estos, se trata de una 'herramienta' nacional que permite al municipio ampliar y mejorar sus servicios sociales hacia la comunidad (Entrevista $n^{\circ} 4$ ). De esta manera, lo que busca el municipio es "brindar a la población lo que necesita de acuerdo a los recursos y herramientas disponibles (...); lo que se divulga no es el programa en sí mismo, sino la Subsecretaría de Empleo" (Entrevista no 4).

En lo tocante al programa Solares, para su implementación se utilizaron los espacios de los Centros Integradores Comunitarios (CIC) del MDS de la nación

\footnotetext{
${ }^{7}$ En reiteradas ocasiones el municipio santafesino ha evitado anunciar el origen jurisdiccional de los programas sociales que implementa, y en caso de hacerlo, suele no dejar en claro en qué consiste el aporte realizado por parte del Estado nacional. En lo que respecta al PJMyMT, esto último se evidencia en el siguiente fragmento emitido por el municipio en alusión a la implementación del PJMyMT: "para articular mejor los esfuerzos entre el ámbito público y privado, se han coordinado las acciones con el MTEySS de la nación, sindicatos, organismos empresariales (...)" (Municipalidad de Santa Fe, 2011: 99.). Asimismo, la información local referida a la política nacional de "Fortalecimiento de las oficinas de empleo" (Resolución No 176/05 del MTEySS de la nación) es escasa o nula.
} 
ubicados en la ciudad. De acuerdo a los actores locales entrevistados, estos centros son concebidos por el municipio como Solares (Entrevista $n^{\circ} 6$ ) y representan parte del escaso aporte que el Estado local recibe del MDS nacional (Entrevistas $n^{\circ} 6$ y 7). En palabras de la coordinadora del programa, la municipalidad no tiene "convenio con el Estado nacional en lo que tiene que ver con Los Solares, [no obstante] sí existen los CIC, que son de gestión común entre el Estado nacional y el municipio" (Entrevista n7).

Más allá de lo señalado por la coordinadora del programa, el Estado local santafesino hizo uso de otras prestaciones de origen nacional. De acuerdo al decreto que anuncia el programa (DMM No 00725/09), algunos de los espacios de los Solares fueron construidos y remodelados por medio de los programas nacionales de Entrenamiento para el Trabajo (Resolución 696/06) y de Empleo transitorio en Obra Pública Local con Aporte de Materiales- "Trabajadores Constructores" (Resoluciones No 1164/06 y No 397/08 del MTEySS).

Además, en los Solares, el municipio ofrece a los vecinos entre sus prestaciones la posibilidad de participar del programa local Huertas Urbanas. Sucede que este último utiliza fondos del PROHUERTA, iniciativa nacional gestionada en los distintos territorios del país a través de acuerdos establecidos entre las administraciones regionales del Instituto Nacional de Tecnología Alimentaria (INTA) y los respectivos municipios. En el caso santafesino, la municipalidad firmó un convenio con el INTA en el año 2009 (registrado en Decreto municipal N01297/2008) en donde ambas partes acordaron realizar "actividades conjuntas destinadas a mejorar las condiciones de los habitantes en situación de pobreza, con particular atención a las necesidades alimentarias a través del fomento de la autoproducción de alimentos en huertas familiares y comunitarias" (DMM N01297/2008).

Resulta de interés mencionar que ninguno de los actores entrevistados hizo mención de estas articulaciones al momento de describir el programa Solares. Por el contrario, limitaron la definición de la participación nacional en el programa al uso municipal del espacio físico de los CIC. Es necesario advertir, no obstante, que más allá del uso de estas prestaciones nacionales, la iniciativa y el diseño del programa Solares es de carácter exclusivamente local, y entendemos, por lo tanto, que este refleja una determinada preocupación y capacidad de acción del municipio santafesino para el tratamiento de problemas sociales.

El uso de datos generados durante la implementación del programa y la generación de instancias de diálogo son también características que se identifican en los procesos de gestión generados por el municipio para ejecutar los dos programas analizados. En relación con la conformación de ámbitos de consenso, ello se evidencia 
en la creación del Foro Productivo, de la Mesa de Concertación y del Consejo Económico y Social para el caso del programa JMyMT, y en la creación del ECINA y la colocación de responsables en cada Centro de Desarrollo para el caso del programa Solares.

En términos generales, concluimos el presente apartado sosteniendo que las distintas estrategias municipales dirigidas a afrontar las problemáticas sociales del territorio, específicamente aquellas utilizadas para implementar los programas JMyMT y Solares, constituyeron claros ejemplos de la capacidad de gestión y de intervención del Estado local santafesino en materia social. Consideramos, por lo tanto, que los compromisos asumidos por este último fueron de gran aporte a la gestión de políticas sociales en la localidad. La municipalidad de Santa Fe presentó idoneidad para adaptar su organigrama, programas de gobierno, y herramientas de gestión disponibles -como ser programas nacionales- a la resolución de los problemas sociales identificados. Específicamente, en base a los resultados obtenidos en la investigación entendemos que la capacidad de gestión del municipio estuvo dada por su buena organización interna (acciones organizadas por temáticas y que involucran en ocasiones a más de un sector y programa, elaboración de Planes de Desarrollo como conductores de la gestión, entre otras) y por el acercamiento generado con los ciudadanos (descentralización administrativa y gestión de políticas sociales en Solares y vecinales). Retomando a García Delgado (1997), podemos afirmar que la municipalidad de Santa Fe se configura como un ente activo que aportó al diseño e implementación de la política social nacional para constituir de esta manera "un cóctel de programas nacionales con propios" (García Delgado 1997: 21).

\section{Conclusiones finales}

A modo de recapitulación, concluimos el presente trabajo afirmando que la municipalidad de Santa Fe representa un caso concreto de refuncionalización de los Estados locales argentinos; ello se debe a que, como se demostró a lo largo del artículo, durante el período 2008-2013, el ente local incorporó a sus funciones iniciales y responsabilidades administrativas otras de carácter económico y social. Esto último se evidencia en los indicadores presentados a lo largo del artículo que dan cuenta de la preocupación del municipio santafesino en la implementación de políticas sociales propias y nacionales. La exposición de objetivos de carácter social en sus Planes de Gobierno, la creación y revitalización de consejos y la firma de convenios con otros niveles del Estado constituyen grandes ejemplos. Estos avances en los procesos de institucionalización de la política social de la municipalidad de Santa Fe también se manifiestan en las variadas estrategias de implementación utilizadas por el Estado local para la ejecución del programa social nacional JMyMT y el programa social local Los 
Solares. En cuanto al uso de prestaciones nacionales por parte del municipio en la oferta de programas sociales locales, entendemos que fue una estrategia de gestión a la que recurrió la municipalidad de Santa Fe para alcanzar mayores resultados y obtener mejoras sociales en la población. Esta estrategia le permitió al Estado local presentar sus servicios a la comunidad de manera más ordenada y acorde a las situaciones y poblaciones a atender. Sin embargo, consideramos que los casos en donde se han utilizado prestaciones nacionales y se ha omitido tal información a la comunidad constituyen, además de una estrategia de gestión, una estrategia política. Esta última le permitiría al municipio sacar rédito político de los mecanismos de articulación intergubernamental existentes puesto que es el Estado local y no el nacional quien obtiene legitimidad en la implementación de las políticas sociales. De igual manera, más allá de las características de las estrategias de gestión utilizadas, queda de manifiesto en el presente artículo la importancia que tuvo el accionar del Estado local santafesino en la etapa de ejecución de políticas sociales nacionales y propias.

Como segunda conclusión del presente trabajo, afirmamos la existencia de una clara y respetada división de tareas entre la municipalidad de Santa Fe y el MTEySS de la nación, y un elevado grado de autonomía decisional por parte de la primera al momento de implementar el programa JMyMT. Como demostramos a lo largo del artículo, esta cordial articulación se sustentó en una abundante normativa de carácter nacional y municipal y fue alterada únicamente en situaciones puntuales.

Por último, a partir de las afirmaciones realizadas hasta el momento, exponemos como tercera conclusión del presente artículo la necesidad de problematizar y mejorar los procesos de articulación. Entendemos que los procesos de articulación constituyen uno de los grandes desafíos perseguido por el Estado en sus distintos niveles y sectores de acción, ya que ninguna instancia local por sí sola y de manera fragmentada es capaz de resolver los problemas sociales del territorio. En el caso particular de la ciudad de Santa Fe, más allá de los avances presentados por el Estado nacional en la materia como la creación de Planes nacionales integrales y la implementación de políticas territoriales-, entendemos que es necesario promover mayores instancias de articulación intergubernamental en la ejecución de la política social. De manera concreta, consideramos que se deben consolidar los procesos de articulación ya existentes y fomentar la instauración de nuevos, específicamente entre la municipalidad y el MDS de la nación.

A modo de cierre, en el presente artículo sostenemos que es necesaria la existencia de municipios fuertes y que no trabajen de manera aislada. Los resultados de 
De Prácticas y discursos/ Universidad Nacional del Nordeste/ Centro de Estudios Sociales

Año 4, Número 5, Julio-Diciembre, 2015

ISSN 2250-6942

esta investigación pueden constituirse en un punto de partida para seguir profundizando en esta necesaria relación. 


\section{Bibliografía}

Andrenacci, L. (2001). La política social de los gobiernos locales en la región metropolitana de Buenos Aires. Ponencia presentada en IV Congreso Internacional del CLAD sobre Reforma del Estado y de la Administración Pública. Buenos Aires, Argentina.

Badía, G., Carmona, R. (2008). La Gestión local en Argentina: situación y perspectivas. Introducción. Buenos Aires: Editorial Los Polvorines. Universidad Nacional de General Sarmiento.

Cao, H. (2008). La administración pública argentina: nación, provincias y municipios. Ponencia presentada en XIII Congreso Internacional del CLAD sobre la Reforma del Estado y de la Administración Pública, Buenos Aires, Argentina.

Cormick, H. (1996). El municipio del conurbano bonaerense. ¿Es posible el paso de la administración a las políticas activas? Revista APORTES para el Estado y la Administración Gubernamental. 8: 1-11.

Costamagna, P. (2007). Políticas e instituciones para el desarrollo económico territorial. El caso de Argentina. Serie Desarrollo Territorial. Área de Gestión del Desarrollo Local y Regional de la CEPAL 1: 5-51.

García Delgado, D. (1997). Hacia un nuevo modelo de gestión local. Municipio y Sociedad Civil en Argentina. Buenos Aires: FLACSO - UBA - UCC.

Hintze, S. (2003). Estado y políticas públicas: acerca de la especificidad de la gestión de políticas para la economía social y solidaria. Ponencia presentada en Segundo Congreso Argentino de Administración Pública. Sociedad, Estado y Administración, Argentina.

Madoery, O. (2011). Más y Mejor Trabajo para todos: del Programa Jefes de Hogar al Programa Jóvenes. Políticas activas de empleo, Argentina 2003-2010. Sistematización y análisis integrado. Programa CEA-OIT, $1^{a}$ ed. Buenos Aires: Oficina de País de la OIT para la Argentina.

Oszlak, O. (2001). El mito del Estado mínimo: una década de reforma estatal en Argentina. Ponencia presentada en el primer Congreso Argentino de Administración Pública: Rosario agosto-septiembre. 
Repetto, F. y Potenza Dal Masetto (2003). Problemáticas institucionales de la política social Argentina: más allá de la descentralización. Ponencia presentada en VIII Congreso Internacional del CLAD sobre la Reforma del Estado y de la Administración Pública, Panamá, 28-31 Oct. 2003.

Repetto, F., Potenza Dal Masetto, F. (2011). Protección social en la Argentina. Serie Políticas Sociales. División de Desarrollo Social de la CEPAL 174:5-55

\section{Publicaciones oficiales}

Municipalidad de Santa Fe (2008) Plan de Desarrollo Santa Fe Ciudad.

Municipalidad de Santa Fe (2009) Cuenta de Inversión.

Municipalidad de Santa Fe (2010) Cuenta de Inversión.

Municipalidad de Santa Fe (2011) Cuenta de Inversión.

Municipalidad de Santa Fe (2011). La Gestión del Cambio 2007/2011.

Subsecretaría de Empleo (2013) Documento Base de Análisis Territorial.

Secretaría Nacional de la Niñez, Adolescencia y Familia (SENAF) del MDS de la nación (2010). S/N.

Ministerio de Trabajo, Empleo y Seguridad Social de la Nación (2010). "Trabajo y Empleo en el Bicentenario. Cambio en la dinámica del empleo y la protección social para la inclusión. Período 2003 - 2010"

\section{Discursos}

Barletta, Mario (2007) Discurso de asunción.

Barletta, Mario (2008) Discurso de cierre del primer año de gestión.

Barletta, Mario (2009) Discurso de Apertura Sesiones Ordinarias del Honorable Concejo Municipal (HCM).

Barletta, Mario (2010) Discurso de Apertura Sesiones Ordinarias del Honorable Concejo Municipal (HCM).

Corral, José Manuel (2011) Discurso de asunción. 
Corral, José Manuel (2012) Discurso de Apertura Sesiones Ordinarias del HCM.

Corral, José Manuel (2013) Discurso de Apertura Sesiones Ordinarias del HCM.

Corral, José Manuel (2014) Discurso de Apertura Sesiones Ordinarias del HCM.

\section{Sitios web visitados}

www.argentina.gob.ar (Presidencia de la Nación).

www.trabajo.gob.ar (MTEySS de la nación).

www.indec.mecon.ar (Instituto Nacional de Estadísticas y Censos- INDEC).

www.santafe.gov.ar (Instituto Provincial de Estadísticas y Censos- IPEC).

www.santafeciudad.gov.ar_(Municipalidad de Santa Fe).

www.ellitoral.com (Diario el Litoral, Santa Fe).

\section{Normativa consultada}

\section{- Nacional:}

Resolución n²56/03 del MTEySS. Se crea el Plan Integral para la Promoción de Empleo

Resolución n 316/05 de la Secretaría de Empleo del MTEySS de la nación. Se crea la Red Federal de Servicio de Empleo.

Resolución n¹76/05 del MTEySS de la nación. Reglamento operativo de la Resolución no 316/05. Se crea la Unidad de Servicio de Empleo en el ámbito de la Dirección Nacional del Servicio Federal de Empleo de la Secretaría de Empleo.

Convenio Marco 96/06 entre el MTEySS y la Municipalidad de Santa Fe para desarrollar de manera permanente estrategias locales de promoción del empleo en el territorio municipal.

Resolución n³36/06 del MTEySS. Se anuncia el programa de Seguro de Capacitación y Empleo (SCyE).

Protocolo Adicional $n^{\circ} 1$ al Convenio Marco 96/06. El MTEySS de la nación y la municipalidad de Santa Fe se comprometen a brindar asistencia técnica para fortalecer 
las capacidades de la Oficina de Empleo y garantizar la implementación del programa Seguro de Capacitación y Empleo.

Convenio n83 entre Secretaría de Empleo del MTEySS y la Municipalidad de Santa Fe. Objetivo: ambas partes se comprometen a ejecutar las acciones y cumplir las prestaciones a su cargo para el fortalecimiento de la Oficina de Empleo Municipal.

Resolución n 497/08 del MTEySS. Se anuncia el Programa Jóvenes con Más y Mejor Trabajo.

Resolución no 261/08 de la SE del MTEySS Reglamento operativo de la Resolución anterior. Anexos I, II, III y IV.

Protocolo Adicional $n^{\circ} 8$ al Convenio n 96/06 entre el MTEySS y la municipalidad de Santa Fe, para la implementación del Programa Jóvenes con Más y Mejor Trabajo.

Resolución n 1164/06 del MTEySS. Creación Programa Nacional de Empleo transitorio en Obra Pública Local con Aporte de Materiales- "Trabajadores Constructores" (Resolución N 1164/06 del MTEySS).

Resolución n 696/06 del MTEySS. Creación programa de Entrenamiento para el Trabajo.

\section{- Municipal:}

Ordenanza n¹1217/05 del Honorable Concejo Municipal de Santa Fe. Se crea la creación de la Oficina de Empleo Municipal.

Decreto n813/05 de la Secretaría de Promoción Comunitaria de la Municipalidad de Santa Fe. Reglamento operativo de la Ordenanza n¹1217/05.

Formulario de Solicitud para la Implementación del Programa Jóvenes con Más y Mejor Trabajo en la ciudad de Santa Fe, presentado por la municipalidad de Santa Fe al MTEySS (2008).

Ordenanza n 11513/08. Se crea la Descentralización Administrativa de la municipalidad de Santa Fe y la conformación de ocho Distritos.

Decreto $n^{\circ}$ 01533/2010 para registrar y enumerar el Protocolo Adicional $n^{\circ} 8$ al Convenio Marco 96/06 firmado por la municipalidad de Santa Fe y el MTEySS. 
Ordenanza no 11.437/07. "Funciones Secretarías", sancionada en noviembre 2007 y promulgada en diciembre 2007. Se publica en Boletín Municipal n 208, en enero 2008.

Decreto $\mathrm{n}^{\circ}$ 01275/08 de la organización funcional del Departamento Ejecutivo Municipal.

Ordenanza no 11829/2012. Nuevas modificaciones en el organigrama funcional municipal.

Decreto n00725/09. Creación Programa Solares.

Decreto n 01297/09. Registra convenio firmado con el INTA.

Ordenanzas n 11,112/04 y n 11,470/09. Creación del Consejo de la Infancia y la Niñez.

Decreto nº 00172/12. Creación Gabinete Social.

Ordenanza n 11362. Creación Consejo Consultivo de Mujeres.

Ordenanza n 11107. Creación consejo de Adultos Mayores.

Decreto nº 00466/09. Creación Consejo Económico y Social.

Decreto n 02187/09. Creación programa de Trabajo y Empleo.

Decreto n00295/12. Creación programa Oficina de Empleo.

Decreto nº 01948/13. Creación programa Calles Seguras e Infancias Protegidas

Ordenanza no 11830/11. Creación Agencia de Cooperación, Inversiones y Comercio Exterior.

Resolución del HCM no 13189. Convocatoria para realizar Audiencia Pública que coloque en consideración de la sociedad civil la temática vinculada a la política social de la ciudad.

\section{Entrevistas realizadas}

A referentes nacionales:

Entrevista $n^{\circ}$ 1: funcionarias de la Gerencia de Empleo y Capacitación Laboral (GECAL) del MTEySS de Santa Fe, y referentes del programa nacional JMyMT. 
Entrevista $n^{\circ}$ 2: Gerenta de la GECAL del MTEySS de la nación de Santa Fe.

Entrevista n³: responsable del Centro de Referencia del Ministerio de Desarrollo Social de la nación (MDS) cd Santa Fe.

- $\quad$ A referentes locales:

Entrevista n4: Directora Ejecutiva del Programa Oficina de Empleo de la Municipalidad de Santa Fe.

Entrevista ${ }^{\circ}$ 5: personal de carrera de la Oficina de Empleo municipal.

Entrevista n6: actual Secretario de Desarrollo Social de la Municipalidad de Santa Fe.

Entrevista n07: Directora Ejecutiva del programa municipal Los Solares.

Entrevista n8: integrante del equipo del programa Solares de la Subsecretaría de Acción Social de la Municipalidad de Santa Fe. 